\title{
Optimizing a Measure of Consistency for Sequence-Space Synaesthesia
}

\author{
Jamie Ward \\ School of Psychology, University of Sussex, Brighton, UK \\ E-mail : jamiew@sussex.ac.uk
}

\begin{abstract}
Current diagnostic measures of sequence-space synaesthesia involve some measure of within-item consistency when placing stimuli (e.g., days, months, numbers) in space. The test developed by Rothen et al. (2016) involves choosing xy locations on a 2D computer screen with three repetitions, and consistency is calculated as the area of the triangle bounded by these three coordinates. The aim of this study is to optimize this measure further. Specifically, a fixed cut-off measure of consistency tends to favour synaesthetes with smaller or simpler spatial forms (because the spatial coordinates are themselves less variable) and disadvantages those with larger or more complex forms. A solution, adapted from Root et al. (2021), was used in which subject-specific cut-offs are used from permuted datasets (with significant consistency defined as $z$ scores $<-2$ ). This was found to improve the sensitivity of the test without compromising on the specificity. In an undergraduate sample, we estimate the prevalence of this form of synaesthesia using this method as $7.9 \%$. In addition, to refining this method we make available our analysis scripts (in R) and a large dataset of synaesthetic responses $(\mathrm{N}=252)$ for other researchers to make use of.
\end{abstract}

\section{Introduction}

People with sequence-space synaesthesia (SSS) experience sequential concepts such as time (days, months, years) and numbers as arranged in space. These may be linear or complex, 2D or 3D, and located internally or projected externally (e.g., in front of or around the body). They are one of the most common types of synaesthesia with estimates of $14.2 \%$ (Seron, Pesenti, Noel, Deloche, \& Cornet, 1992), 12\% (Sagiv, Simner, Collins, Butterworth, \& Ward, 2006), 4.4\% (Brang, Teuscher, Ramachandran, \& Coulson, 2010), $8.1 \%$ and $12.8 \%$ (Ward et al., 2018). Attempts to diagnose SSS rely heavily on the notion of test-retest consistency, i.e. genuine synaesthetes should experience these sequences in a stable configuration. The first attempts were human judgments of drawings or descriptions produced at two time points (e.g. Sagiv et al., 2006) with subsequent tests developed to capture quantitative data within a single session. These tests required participants to locate labelled points relative to an avatar (Eagleman, 2009) or ones own body (Smilek, Callejas, Merikle, \& Dixon, 2006), or imagine the spatial form projected on to a 2D screen (Brang et al., 2010; Smilek et al., 2006). However, none of these earlier studies provided normative values, or cut-offs, to discriminate synaesthetes from non-synaesthetes.

Rothen et al. (2016) used Receiver Operating Characteristics (ROC) to establish standardized cutoffs using a computerized test. In this test, participants are required to make a mouse click on a computer screen in a position corresponding to a given label for a day, month, or number. Each stimulus is repeated three times so that the within-item consistency could be calculated. Several different ways of calculating 
consistency were compared and the best performing one - i.e. the one that maximally discriminated between synaesthetes and non-synaesthetes - was the area of the triangle bounded by the three xy coordinates. That is, if the three points are close together in xy space then the triangle is smaller in area. Their suggested cutoff was that the average size of the triangle should be less than $0.203 \%$ of the area of the screen in order to qualify as a synaesthete. Ward et al. (2018) showed that the same cut-off was appropriate when the test was migrated from an in-person test with a standard screen size (as in Rothen et al. 2016) to an online environment where it varies but can nevertheless be measured (noting that the test was configured to not work on mobile screens or tablets).

Although a reliance on consistency to diagnose synaesthesia is standard for the field, there are various problems in using it solely to diagnose sequence-space synaesthesia. For example, some nonsynaesthetes can easily imagine numbers in a straight line for the purposes of this task but do not do so in daily life. Other non-synaesthetes may click in the same place on the screen because they don't understand the instructions and achieve a high consistency score by trivial means. To counter these issues, Ward et al. (2018) put various other checks in place and these are shown in the decision tree in Figure 1 (left panel). First of all, a questionnaire was devised whereby participants were asked questions such as whether they visualize sequences outside of the experiment. Responses are summed across Likert questions a score $<=$ 19 is taken as indicative of SSS. Synaesthetes need to pass both the consistency measure and the questionnaire. A second check is to ensure that people who are consistent have clicked on a sufficiently diverse set of $x y$ coordinates on the screen. Specifically, the standard deviation of the x-coordinates and/or the standard deviation of the $y$-coordinates (measured across all trials) should exceed a proposed value of 0.075 for a normalized screen with width and height of 1 unit. A participant who produced a horizontal straight-line form would have a very low standard deviation in the y-coordinates but a high standard deviation in $\mathrm{x}$-coordinates, and a participant with a vertical line would have the reverse profile. A participant with a circular spatial form would be high on both. A participant who clicks randomly around the screen would also be high on both $\mathrm{x}$ and $\mathrm{y}$ standard deviation, but would fail the consistency tests (the triangles would be large).
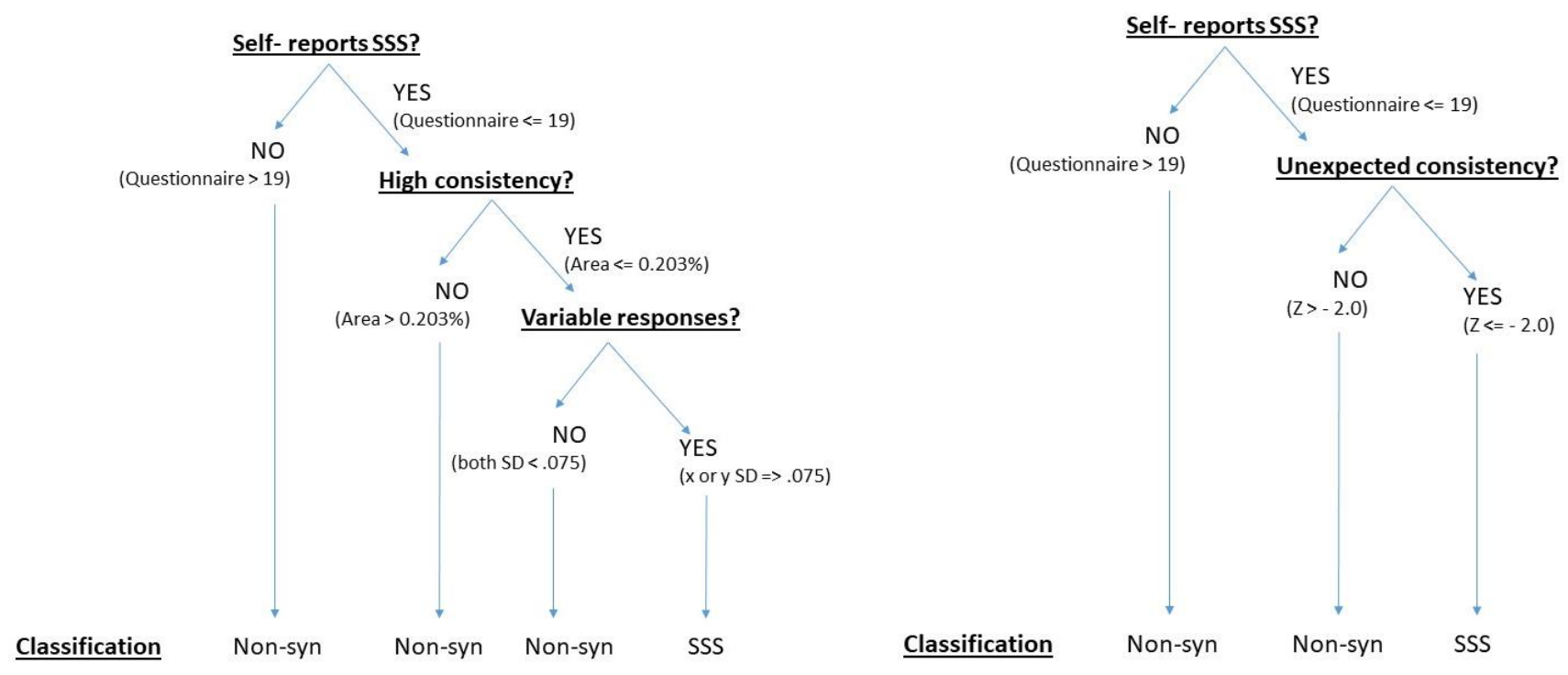
Figure 1: Two alternative ways of diagnosing sequence-space synaesthesia (SSS) based on a test of consistency and a questionnaire measure. The left side is the published method of Ward et al. (2018). This uses absolute cut-off values. The right side is the novel method proposed here. This is based on subjectspecific cut-off values derived from permuted datasets.

One problem with this diagnostic is that it may benefit some synaesthetes more than others. Specifically, it is easier for synaesthetes with straight line spatial forms to achieve higher consistency (smaller areas of triangles) because there is less variability overall. Their responses vary in one-dimension rather than two. In an analagous situation, a synaesthete with a sparse colour palette (e.g. many black or dark colours) would find it easier to pass than someone with a very diverse colour palette. Root et al. (2021) proposed a modified within-subject measure of consistency that deals with this kind of problem in the domain of colour. They calculated colour consistency in the standard way, but then that individual's data is shuffled multiple times to get a within-subject distribution of chance levels of consistency. Unexpectedly high consistency is considered (the upper $5 \%$ of permutations corresponding to $z>2$ ), and not just absolute consistency. In the case of diagnosing SSS, an equivalent z-score would be based on the question: how likely is it that the observed level of consistency could be found based on the observed level of variability in that individual's spatial distribution of coordinates? The classification thresholds for $\mathrm{x}$ - and $\mathrm{y}$ - standard deviation would no longer be necessary and, in effect, would be replaced by a within-subject measure of variability. Moreover, a person who clicks in the same place would get a z-score around zero because high consistency is expected for someone adopting that strategy. A person with more complex forms would automatically be afforded more 'slack' in passing the test than someone with a straight line because each individual would have their own distributions of chance that are not hard-coded by the researcher.

The aim of this study is to compare the original method against this alternative method (both shown in Figure 1) on new datasets recruited from a sample of self-referred sequence-space synaesthetes and a normative sample (who we can assume are mainly non-synaesthetes but will also contain some people with SSS).

\section{Method}

\section{Participants}

There were 252 self-referred sequence-space synaesthetes who completed the test (202 females, 44 males, 6 other; mean age $=37.313$ years, S.D. $=13.845)$. These participants had contacted our research group via our website (www.sussex.ac.uk/synaesthesia) and were subsequently invited to take the test.

There were 215 controls (178 females, 37 males; mean age $=19.902$, S.D. $=5.007$ ) who completed the test as part of course credits at the University of Sussex. Hence, this group tended to be younger.

None of the participants had previously taken part in the earlier studies of Ward et al. (2018) or Rothen et al. (2016). The study was approved by the Cross-Schools Science and Technology Research Ethics Committee at the University of Sussex. 
The 29 stimuli consisted of 12 months of the year, 7 days of the week, and 10 numbers (digits 0 to 9). Each stimulus was presented three times so that there were 87 trials in total. The test was run in Syntoolkit and a demonstration version is available here (https://www.syntoolkit.org/studies/bf-ss-2/practice). This bespoke software is not available for sharing but is easy to implement in any online platform that enables the collection of mouse click coordinates and can access the screen resolution.

\section{Procedure}

For the consistency test, participants were given the following instructions (taken from Ward et al., 2018):

"In this study you will see single digits (e.g. 5), days of the week (e.g. Tuesday), and months of the year (e.g. July) displayed in the centre of the computer screen. Your task is to think about how these concepts may be arranged spatially on the 2D computer screen. Some people may automatically think about these concepts spatially in their everyday life, and you should use this. For other people this may seem like a strange task, but just go with your intuitions. There isn't a right or wrong answer. When you see each item on the computer screen, then visualise where it fits spatially and click the mouse in the corresponding location on the screen (using a left click). Each item is repeated three times."

A number (digits $0-9)$, day $(\mathrm{N}=7)$ or month $(\mathrm{N}=12)$ was presented in the centre of a white screen and remained visible until the participant responded with a mouse click. The next stimulus appeared immediately after the click. Each number, day and month was presented three times. The order of the stimuli was randomised, but such that no stimulus was repeated until the previous batch of unique stimuli $(\mathrm{N}=29)$ had been presented.

The questionnaire appeared immediately after the consistency test and included the following 13 questions taken from Ward et al. (2018). The answers to the nine questions answered on a Likert scale (i.e. question 1 and questions 5-12) are summed together to give a total score, following reverse coding of four items. The minimum score was 9 (an ideal synaesthete) and the maximum score was 45 (an ideal nonsynaesthete). The remaining questions are not analysed quantitatively but provide additional information about experiences of synaesthesia and the task.

1. Some people routinely think about sequences as arranged in a particular spatial configuration (as in the examples below), do you think this might apply to you? ( $1=$ strongly agree, $5=$ strongly disagree) 

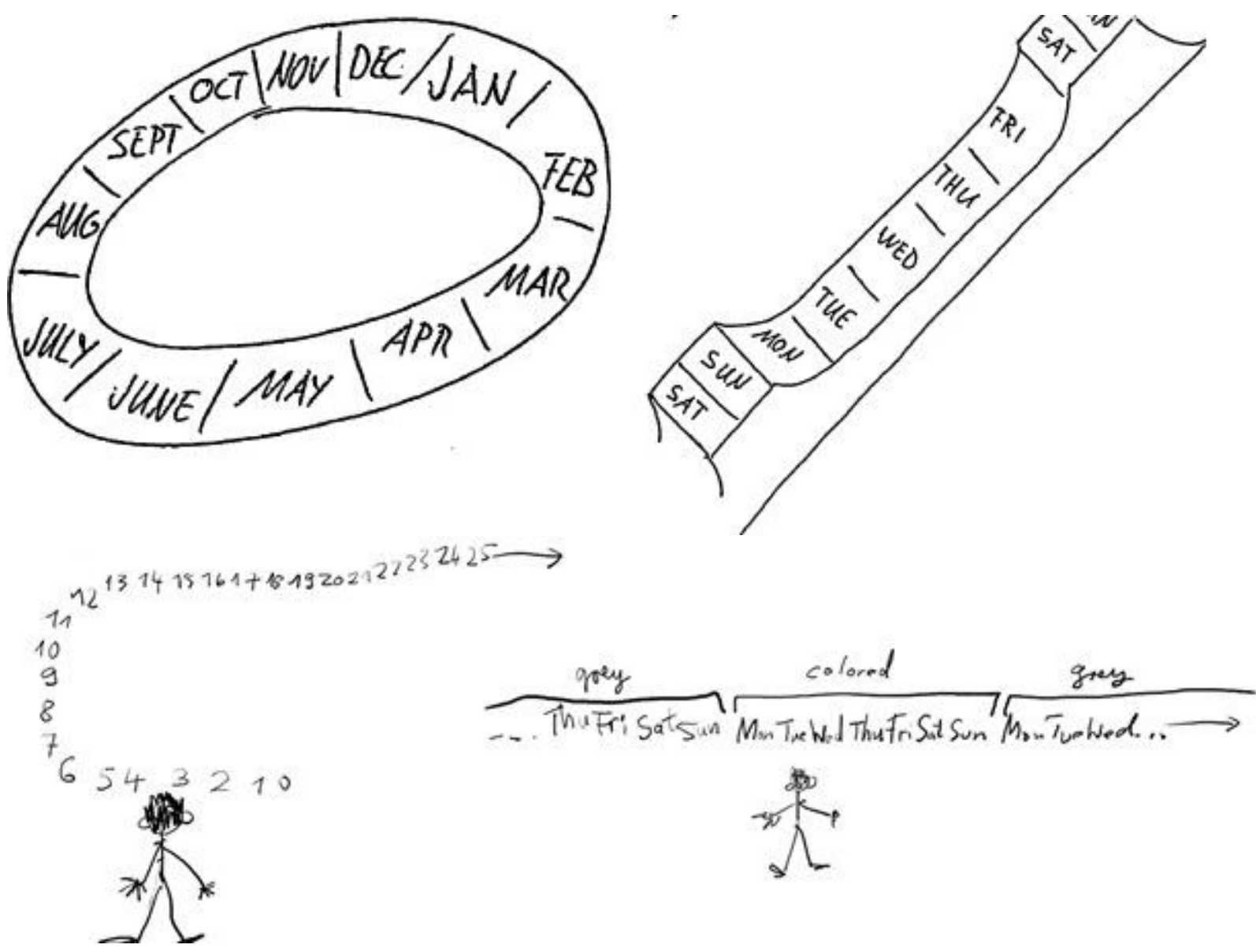

Figure 2: Images accompanying the sequence-space questionnaire.

2. Which of the following do you routinely think about in this way? (Numbers, days, months, years, letters of the alphabet, temperature, height, weight)

3. Where do you tend to routinely experience these sequences? $(1=$ in the space outside my body; $2=$ on an imagined space that has no real location; $3=$ inside my body; $4=$ this doesn't apply to me!)

4. What kind of characteristics do these spatial sequences always tend to take? (Colors; shading; 2D; 3D; perspective; like blocks or tiles; a certain font) [checkbox responses]

5. Before doing this experiment, I always thought about NUMBERS as existing in a particular spatial sequence ( $1=$ strongly agree; $5=$ strongly disagree)

6. Before doing this experiment, I always thought about DAYS OF THE WEEK as existing in a particular spatial sequence ( $1=$ strongly agree; $5=$ strongly disagree)

7. Before doing this experiment, I always thought about MONTHS OF THE YEAR as existing in a particular spatial sequence ( $1=$ strongly agree; $5=$ strongly disagree)

8. I use this way of thinking about spatial sequences in my everyday life ( $1=$ strongly agree; $2=$ strongly disagree)

9. When doing the experiment, I didn't have any strong intuition as to where to put the NUMBERS (1= strongly agree; $5=$ strongly disagree) [Reverse coded]

10. When doing the experiment, I didn't have any strong intuition as to where to put the DAYS OF THE WEEK ( 1 = strongly agree; $5=$ strongly disagree) [Reverse coded] 
11. When doing the experiment, I didn't have any strong intuition as to where to put the MONTHS OF THE YEAR ( $1=$ strongly agree; $5=$ strongly disagree) [Reverse coded]

12. This experiment didn't really make much sense to me ( $1=$ strongly agree, $5=$ strongly disagree) [Reverse coded]

13. Feel free to enter any comments here. E.g. what strategy did you use? Do you want to clarify any of the above answers?

\section{Analyses}

The $\mathrm{R}$ scripts for analyzing the data are included in the OSF repository, and the core data needs to be organized into three columns (data label, $x$-coordinate, $y$-coordinate) and 87 rows (i.e. one row per trial).

\section{Calculating consistency}

Each stimulus is represented by three xy coordinates - $\left(x_{1}, y_{1}\right),\left(x_{2}, y_{2}\right),\left(x_{3}, y_{3}\right)$ - from the three repetitions. For each stimulus, the area of the triangle bounded by the coordinates is calculated as follows:

Area $=\left(x_{1} y_{2}+x_{2} y_{3}+x_{3} y_{1}-x_{1} y_{3}-x_{2} y_{1}-x_{3} y_{2}\right) / 2$

The mean area is calculated by adding together the area for each stimulus and dividing by 29 . This unit is transformed into a percentage area taking into account the different pixel resolution of each participant.

Mean area $=($ Summed area $/ 29) * 100 /$ Screen area

Where: Screen area $=X_{\text {pixels }}{ }^{*} Y_{\text {pixels }}$

\section{Calculating chance levels of consistency}

To create permuted datasets for each participant: the $87 \mathrm{xy}$ coordinates are randomly shuffled so they are no longer linked to the original data labels ("Monday", " 5 ", "April", etc.). The mean area of the triangles based on the shuffled coordinates is computed (as described above), and the whole process is repeated 1000 times to obtain a subject-specific distribution of chance levels of consistency. A z-score is calculated comparing the observed consistency against the mean and SD of the permuted data:

$Z=[($ observed consistency $)-($ mean consistency of permuted data) $]$ / (SD of permuted data)

\section{Results}

\section{Questionnaire scores}

The mean questionnaire score of the sample recruited from the synaesthesia database was 13.599 (S.D. $=4.846)$ and the mean questionnaire score of the sample recruited from the general population was 29.781 (S.D. = 8.566). The distribution is shown in Figure 3. It can be seen from the point of intersection of the two distributions, that the cut-off value of 19 proposed by Ward et al. (2018) appears justified when considering this larger and novel sample. 


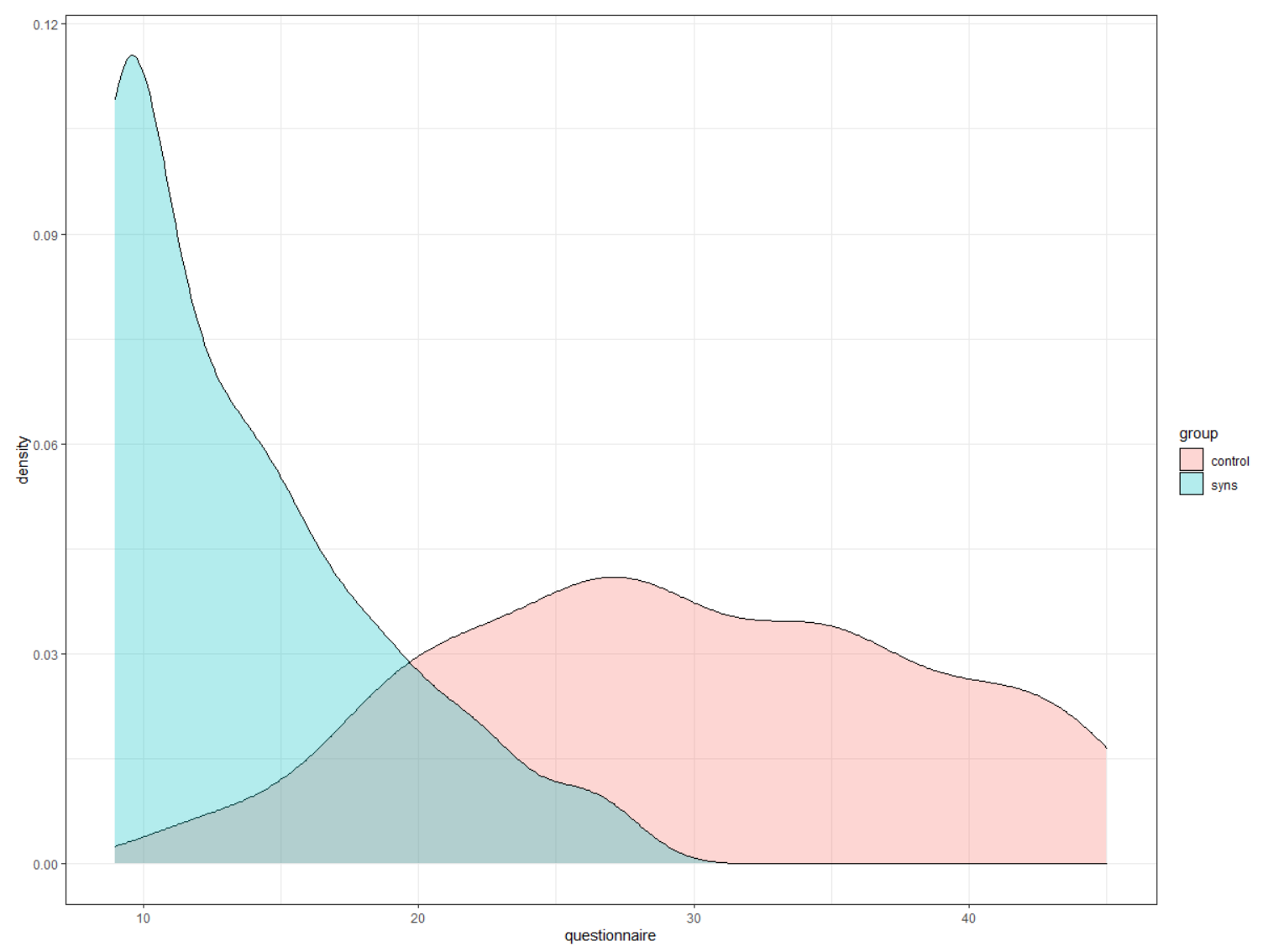

Figure 3. Density plots (such that the area under the curve for each group sums to 1) for synaesthetes (turquoise) and a normative sample (pink) according to their score on the post-test questionnaire.

\section{Comparison of Methods}

Table 1 shows the numbers of participants passing either the consistency test and/or the questionnaire from the two groups. In subsequent analyses, classification as a synaesthete depends on passing both measures.

In the revised method, $81.3 \%$ (205/252) of the synaesthete sample would be classed as synaesthetes which compares to only $67.1 \%(169 / 252)$ using the original method. That is, the sensitivity of the test is significantly increased $\left(X^{2}(1)=13.434, p<.001\right)$.

In the revised method, $7.9 \%(17 / 215)$ of the normative sample would be classed as synaesthetes which compares to $5.1 \%(11 / 215)$ using the original method. This is not significantly different $\left(x^{2}(1)=1.375\right.$, $p=.241)$. These values constitute estimates of the prevalence within this normative sample. As such, we conclude the revised method improves the test sensitivity without compromising on its specificity. 
Table 1: Numbers of participants passing and failing the questionnaire or consistency test from the two groups of participants using the original classification procedure (left) or the revised method (right).

\begin{tabular}{|c|c|c|c|}
\hline \multirow{2}{*}{\multicolumn{2}{|c|}{$\begin{array}{l}\text { Original } \\
\text { Method }\end{array}$}} & \multicolumn{2}{|c|}{ Consistency Test } \\
\hline & & Failed & Passed \\
\hline \multirow{8}{*}{ 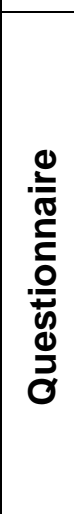 } & & $35.1 \%$ & $12.4 \%$ \\
\hline & Failed & $\mathrm{N}=164$ & $\mathrm{~N}=58$ \\
\hline & & (24 syns, & (10 syns, \\
\hline & & 140 cont) & 48 cont) \\
\hline & & $13.9 \%$ & $38.5 \%$ \\
\hline & Passed & $\mathrm{N}=65$ & $\mathrm{~N}=180$ \\
\hline & & (49 syns, & (169 syns, \\
\hline & & 16 cont) & 11 cont) \\
\hline
\end{tabular}

\begin{tabular}{|c|c|c|c|}
\hline \multirow{2}{*}{\multicolumn{2}{|c|}{$\frac{\text { Revised }}{\underline{\text { Method }}}$}} & \multicolumn{2}{|c|}{ Consistency Test } \\
\hline & & Failed & Passed \\
\hline \multirow{2}{*}{ 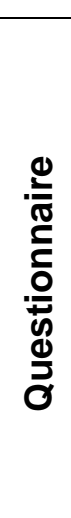 } & Failed & $\begin{array}{c}22.9 \% \\
\mathrm{~N}=107 \\
\text { (4 syns, } \\
103 \text { cont) }\end{array}$ & $\begin{array}{c}24.6 \% \\
\mathrm{~N}=115 \\
\text { (30 syns, } \\
85 \text { cont) }\end{array}$ \\
\hline & Passed & $\begin{array}{c}4.9 \% \\
N=23 \\
\text { (13 syns, } \\
10 \text { cont) }\end{array}$ & $\begin{array}{c}47.5 \% \\
N=222 \\
\text { (205 syns, } \\
17 \text { cont) }\end{array}$ \\
\hline
\end{tabular}

An extra 46 participants (39 synaesthetes, 7 controls) who didn't originally meet the criteria for SSS, would now do so and 4 participants (3 synaesthetes, 1 control) previously classed as SSS would be classed as non-synaesthetes. To understand what these cases look like, we can visualize the data. A java-based data visualizer is included in this OSF for displaying this data.

Figure 4 (top) shows a case of someone with a consistency score of 0.266 which is higher than the 0.203 cut-off and previously would be classed as a fail. However, the mean expected consistency by chance is 5.489 (S.D. $=0.888$ ) giving a z-score of -5.879 . The fact that the participant selected a diverse set of $x y$ locations counts in their favour. The case in the middle is similar but the failure was driven almost entirely by one large inconsistency (for the number 9) which inflated the mean area. The third case initially failed for another reason: the standard deviations of the $x$ and $y$ positions were below the threshold ( 0.06 and 0.07 ), because their responses occupy a small region of space and there is (presumably) no spatial sequence for numbers. However, the z-score for this participant is $\mathbf{- 5 . 8 0 8}$ and they pass under the revised criteria. 


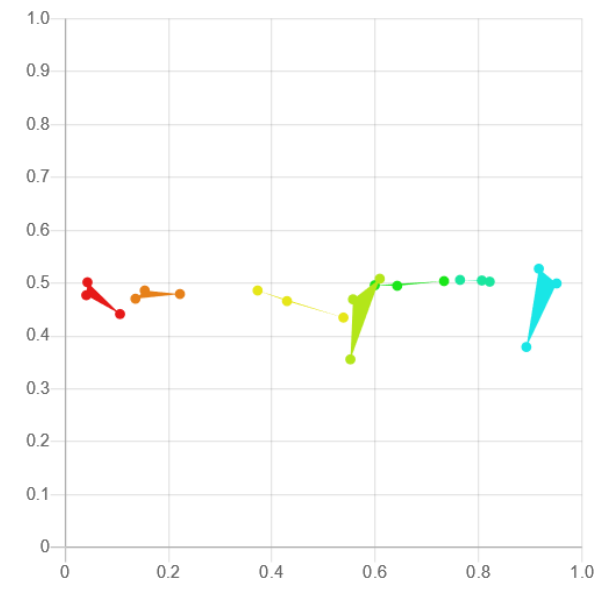

Monday Tuesday Wednesday Thursday - Friday Saturday $\bullet$ Sunday

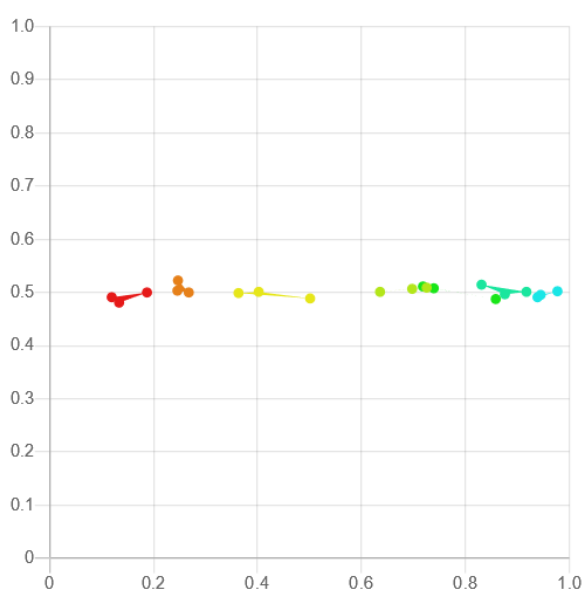

- Monday Tuesday Wednesday Thursday - Friday Saturday Sunday

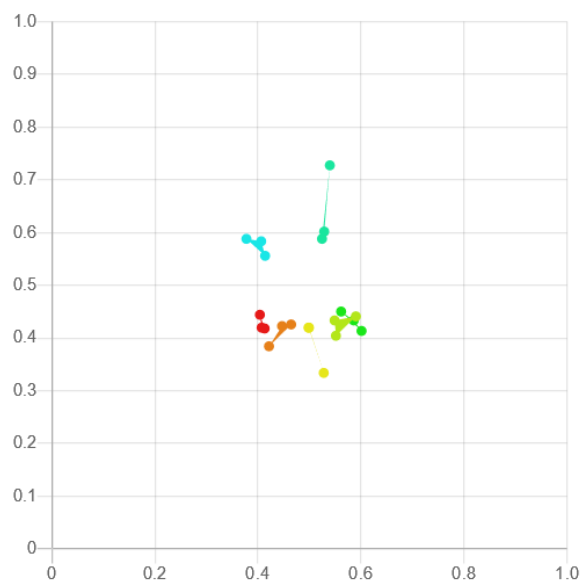

- Monday - Tuesday $\odot$ Wednesday $\bullet$ Thursday - Friday Saturday Sunday
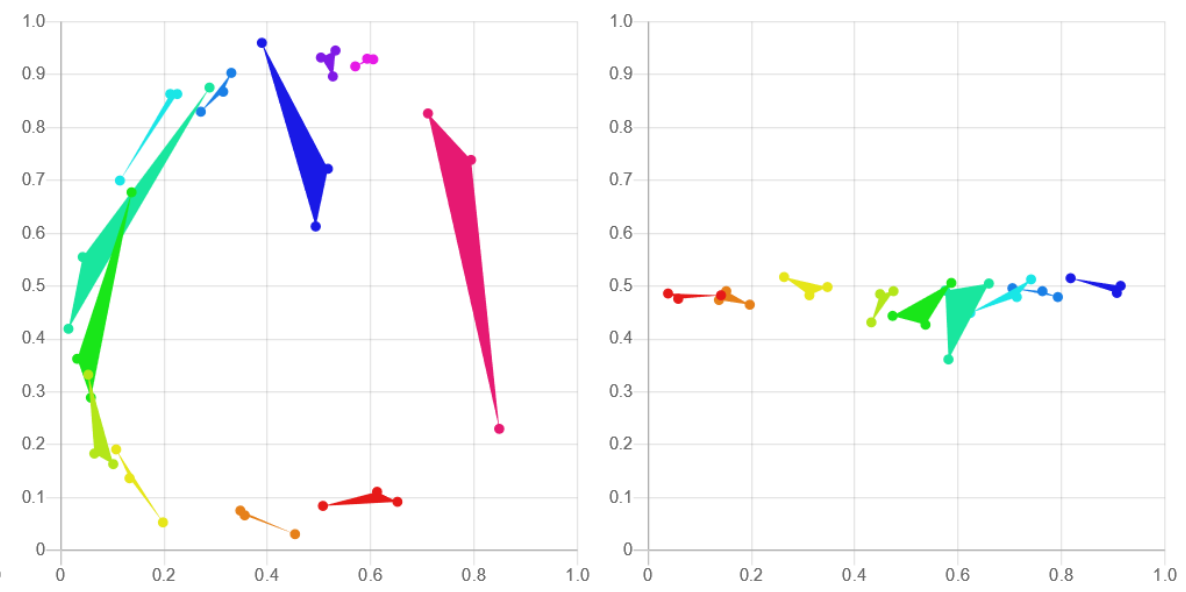

January February March April May - June July August $\bullet$ September $\bullet$ October November December
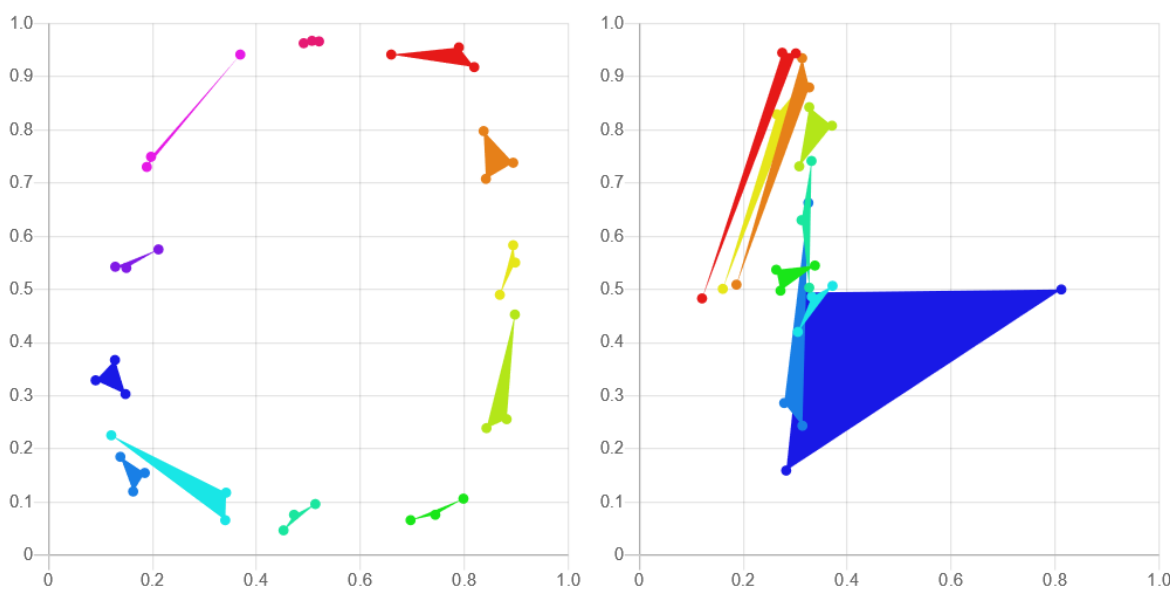

- January - February March $\bullet$ April $\bullet$ May - June July August $\bullet$ September $\bullet$ October - November December
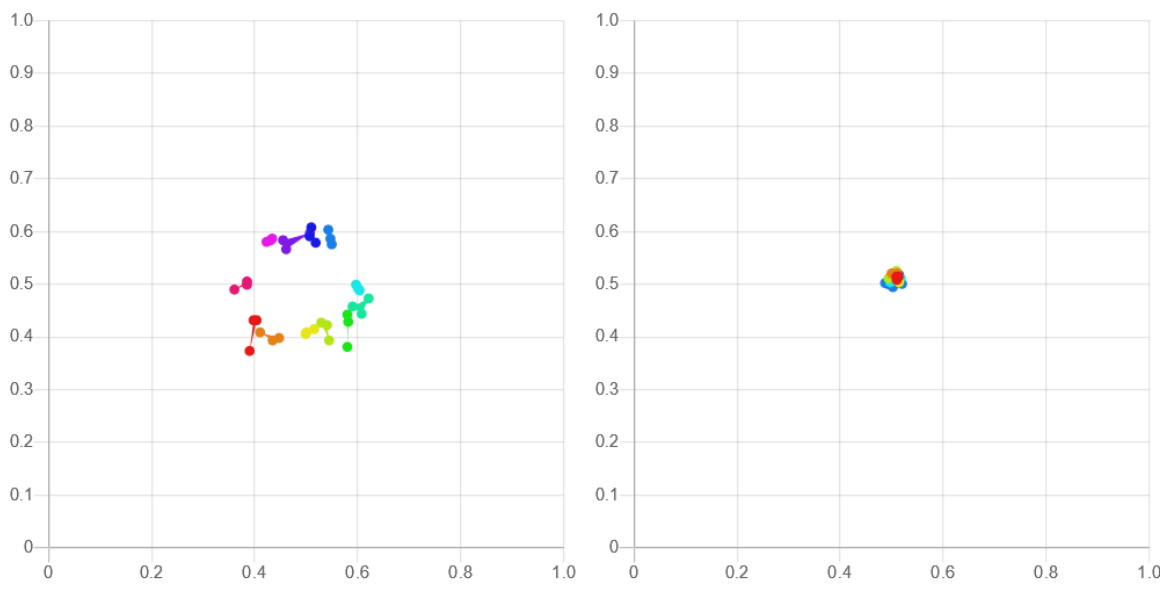

- January - February March April - May June July August $\bullet$ September $\bullet$ Octobe November December

Figure 4: Example spatial forms from three participants (top, middle, bottom) who self-report sequence space synaesthesia. These participants were initially classed as failing the test of consistency (with the criteria of Ward et al., 2018) but would pass it using revised criteria (adapted from Root et al., 2021). 
Finally, two examples of cases who initially passed but now fail are shown in Figure 5 . By visual inspection, these cases would not be convincing examples of SSS. The general feature of all of these cases is that the mouse position shifted between a small number of discrete screen locations. This is sufficient to generate consistency and variability but, when modelled as z-scores, the results are shown to be explicable by chance alone ( $z$ of -1.452 and -1.136 respectively).

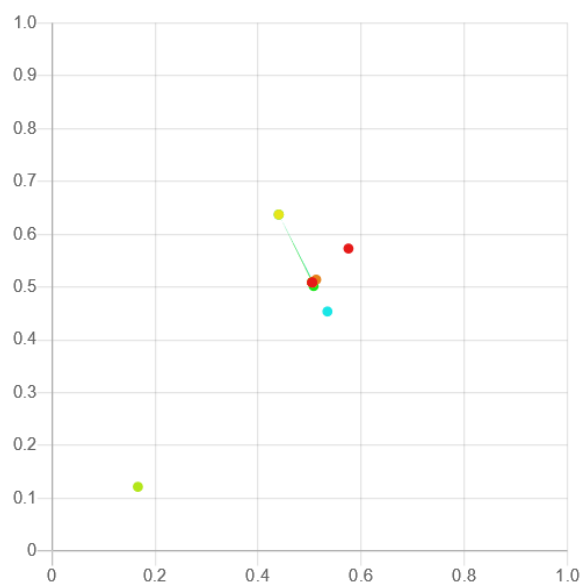

- Monday - Tuesday Wednesday - Thursday - Friday Saturday Sunday

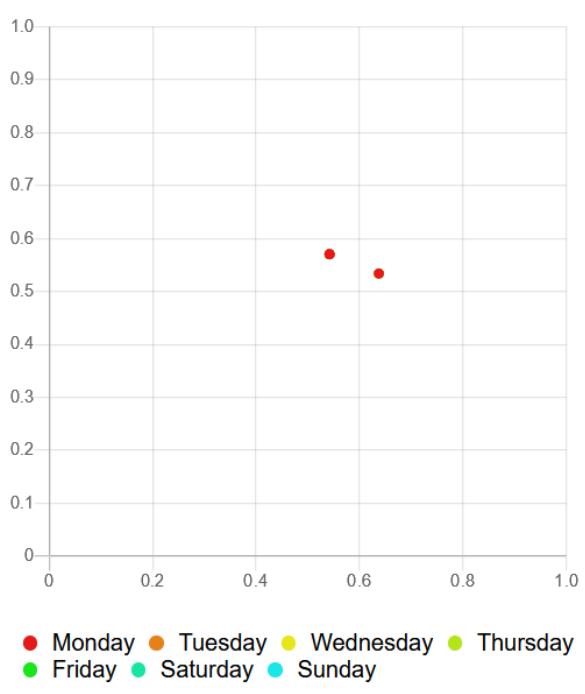

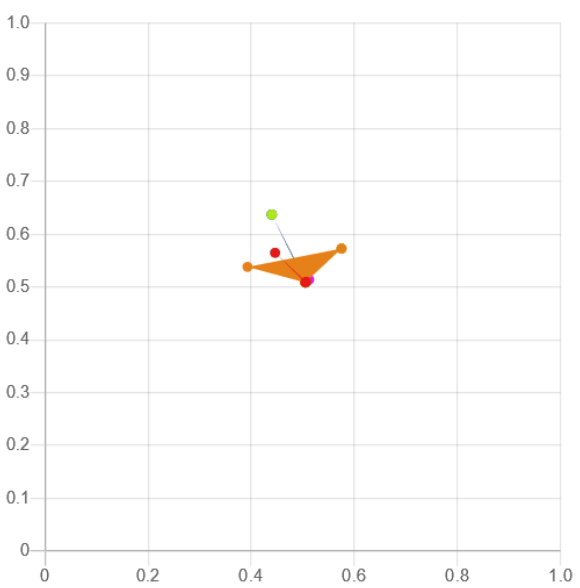

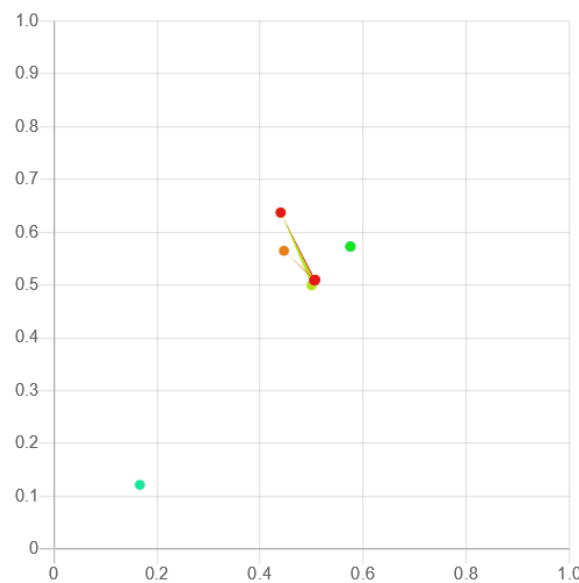

January - February March April May June July $\bullet$ August $\bullet$ September $\bullet$ Octobe November December

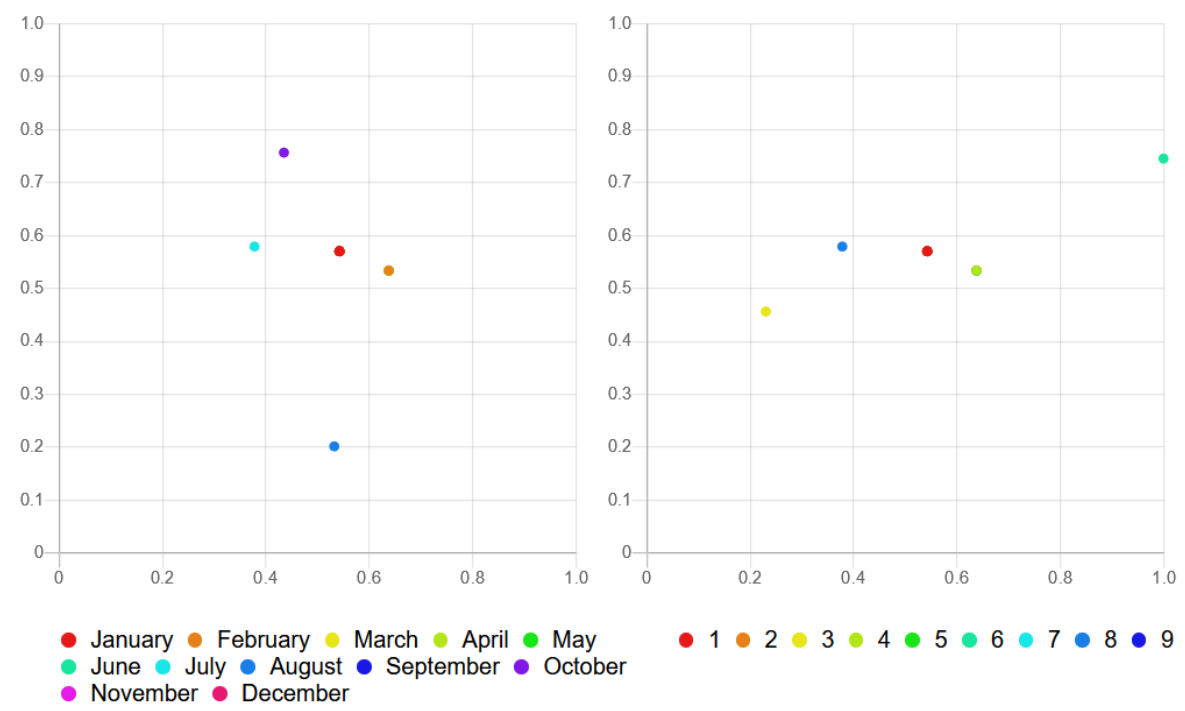

Figure 5: Example spatial forms from two participants (top, middle, bottom) who were initially classified as passing the consistency test (with the criteria of Ward et al., 2018) but would fail it using revised criteria (adapted from Root et al., 2021).

\section{Further Exploratory Analyses of the Revised Method}

The previous analyses relied on a consistency cut-off of -2 standard deviations, and a questionnaire cut-off $<=19$. This section considers how robust the results are to changes in these values. It also considers whether other options fare better. 
The mean z-score of the synaesthetes was -5.032 (S.D. = 1.666) and the mean z-score of the controls was -1.789 (S.D. = 3.322). In general, synaesthetes are far more homogeneous in their approach to this task whereas others show considerable heterogeneity as shown in Figure 6.

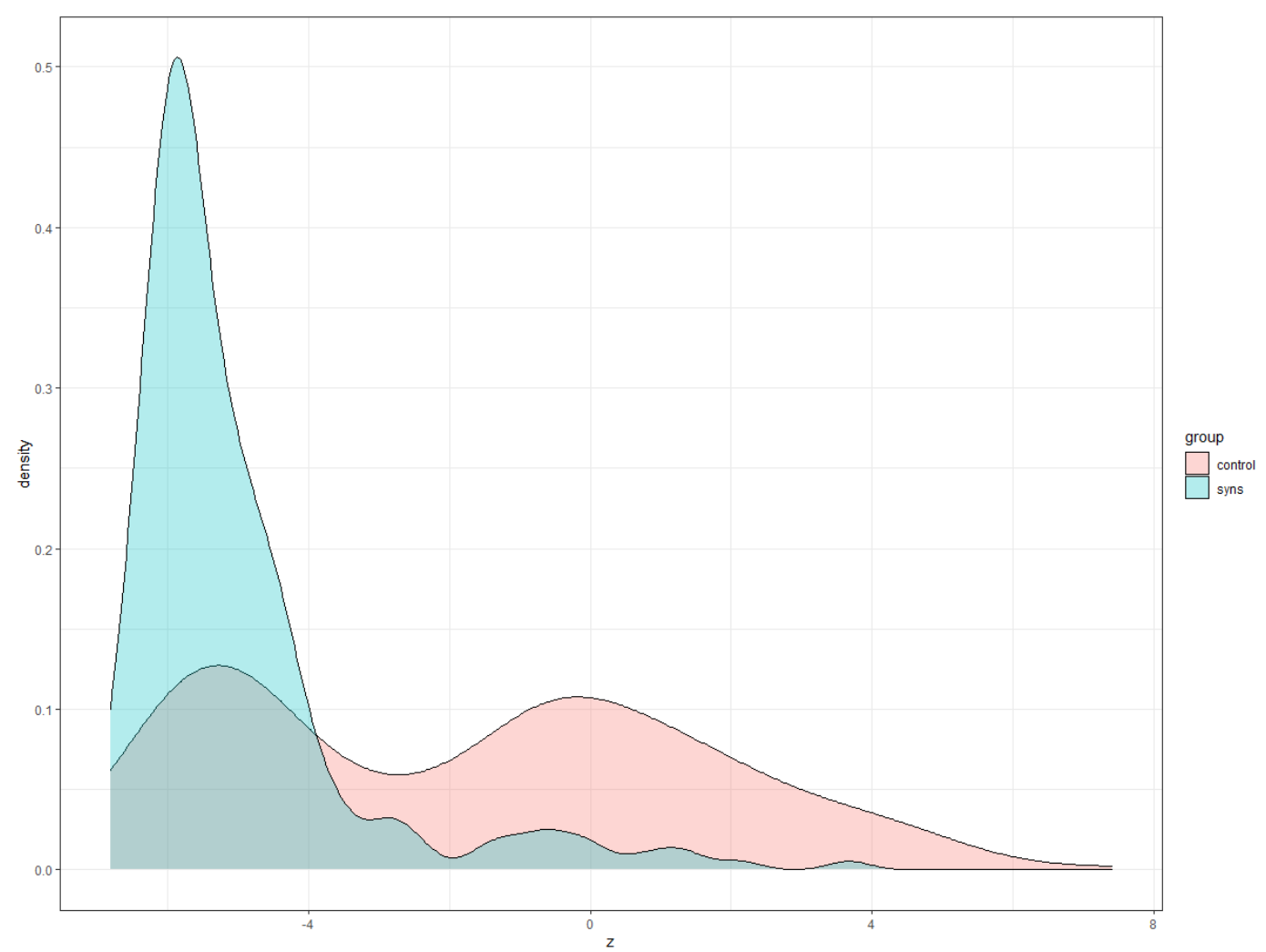

Figure 6. Density plots (such that the area under the curve for each group sums to 1) for synaesthetes (turquoise) and a normative sample (pink) according to their consistency score (z-scores relative to their permuted dataset).

Table 2 shows the different sensitivity, specificity, and overall accuracy of the revised classification for different z-score consistency cut-offs and different questionnaire scores. Changing the questionnaire cut-offs tends to have larger effects on the classification than changes to the z-score cut-off. However, the new method is more sensitive at detecting synaesthetes than the original method of Ward et al. (2018) no matter what the cut-off values are (at least within the range considered here). The optimal accuracy occurs for a questionnaire cut-off of $<=19$ and a consistency cut-off of $z<-1.5$ although it is virtually identical to $z<$ -2.0 . 
Table 2: Sensitivity, specificity and accuracy (top, middle, bottom tables) as a function of varying the questionnaire cut-off (rows) and consistency cut-off (columns). The values on the right side show the values of sensitivity, specificity and accuracy for this dataset when applying the published cut-offs used in Ward et al. (2018).

\section{Sensitivity (Prob of classing syn as a syn)}

\begin{tabular}{l|r|r|r|r|r|r|r|} 
& $\mathbf{- 4 . 5}$ & $\mathbf{- 4}$ & $\mathbf{- 3 . 5}$ & $\mathbf{- 3}$ & $\mathbf{- 2 . 5}$ & $\mathbf{- 2}$ & $\mathbf{- 1 . 5}$ \\
\hline$<=17$ & 0.679 & 0.734 & 0.746 & 0.746 & 0.754 & 0.754 & 0.758 \\
\hline$<=18$ & 0.702 & 0.762 & 0.774 & 0.774 & 0.782 & 0.782 & 0.786 \\
\hline$<=19$ & 0.730 & 0.790 & 0.802 & 0.802 & 0.810 & 0.813 & 0.817 \\
\hline$<=20$ & 0.734 & 0.802 & 0.813 & 0.813 & 0.829 & 0.833 & 0.837 \\
\hline$<=21$ & 0.750 & 0.821 & 0.837 & 0.837 & 0.853 & 0.857 & 0.861 \\
\hline
\end{tabular}

Specificity (Prob of classing control as a control)

\begin{tabular}{|c|c|c|c|c|c|c|c|c|}
\hline & -4.5 & -4 & -3.5 & -3 & -2.5 & -2 & -1.5 & Original specificity \\
\hline$<=17$ & 0.977 & $0.977^{\prime}$ & $0.977^{\prime}$ & $0.972^{\prime}$ & $0.972^{\prime}$ & 0.972 & 0.972 & 0.949 \\
\hline$<=18$ & $0.953^{\prime}$ & $0.949^{\prime}$ & $0.949^{\prime}$ & $0.944^{\top}$ & $0.944^{\top}$ & 0.944 & 0.944 & \\
\hline$<=19$ & $0.935^{\prime}$ & $0.926^{\prime}$ & $0.926^{\prime}$ & $0.921^{\top}$ & $0.921^{\top}$ & 0.921 & 0.921 & \\
\hline$<=20$ & $0.916^{\prime}$ & $0.907^{\prime}$ & $0.907^{\prime}$ & $0.902^{\prime}$ & $0.898^{\prime}$ & 0.898 & 0.898 & \\
\hline$<=21$ & $0.898^{\prime}$ & $0.884^{\prime}$ & $0.884^{\top}$ & $0.879^{\prime}$ & $0.874^{\top}$ & 0.874 & 0.874 & \\
\hline
\end{tabular}

Accuracy (mean of sensitivity+specificity)

\begin{tabular}{|l|r|r|r|r|r|r|r|r|}
\hline & $\mathbf{- 4 . 5}$ & $\mathbf{- 4}$ & $\mathbf{- 3 . 5}$ & $\mathbf{- 3}$ & $\mathbf{- 2 . 5}$ & $\mathbf{- 2}$ & $\mathbf{- 1 . 5}$ & Original accuracy \\
\hline$<=17$ & 0.828 & 0.855 & 0.861 & 0.859 & 0.863 & 0.863 & 0.865 & 0.81 \\
\hline$<=18$ & 0.828 & 0.855 & 0.861 & 0.859 & 0.863 & 0.863 & 0.865 & \\
\hline$<=19$ & 0.833 & 0.858 & 0.864 & 0.861 & 0.865 & 0.867 & 0.869 & \\
\hline$<=20$ & 0.825 & 0.854 & 0.860 & 0.858 & 0.864 & 0.866 & 0.867 & \\
\hline$<=21$ & 0.824 & 0.853 & 0.861 & 0.858 & 0.864 & 0.866 & 0.868 & \\
\hline
\end{tabular}

\section{Discussion}

Sequence-space is one of the most common types of synaesthesia - here the estimate is $7.9 \%$ of the population. As such it could be an attractive type of synaesthesia for researchers to study in order to recruit sufficiently large samples. However, establishing a standard test for SSS and a standard diagnostic cut-off has proved slower and more difficult than for grapheme-colour. On tests of personality and cognition, people with SSS are at least as different from neurotypicals as grapheme-colour (Mealor, Simner, Rothen, Carmichael, \& Ward, 2016; Ward, Rothen, Chang, \& Kanai, 2017), thus its higher prevalence is not necessarily indicative of it being a 'weaker' form of synaesthesia.

One of the challenges in devising a diagnostic test is to balance the need to make it fair to synaesthetes (high sensitivity) without making it too easy for non-synaesthetes to pass. A deeper issue is that there is no ground-truth for knowing who does and does not have synaesthesia in the first place. Here, our candidate synaesthetes were people who had contacted our research group and declared having SSS and most of this sample subsequently reconfirmed having this type of synaesthesia on a later date when 
given a questionnaire (Figure 3). However, the cut-off norms provided by Ward et al. (2018) were arguably too conservative (with only $67 \%$ of that sample passing). Instead, using a cut-off based on subject-specific z-scores significantly improved that figure. In this approach, the consistency of each person (still measured by the area of the triangle bound by three xy coordinates) is compared against consistency measures that could have been obtained by chance (by randomly shuffling the same xy coordinates). This approach can easily reject participants who repeatedly click in the same/similar locations irrespective of stimulus (because the chance distribution predicts high consistency) and, at the same time, is more generous towards participants with spatial forms that are more elaborate in nature (because the chance distribution predicts low consistency). Root et al. (2021) also found that this was a good way of excluding likely non-synaesthetes because they had insufficient colour variability, and we suggest that this general principle may be helpful for all other kinds of diagnostic tests of synaesthesia based on consistency.

\section{References}

Brang, D., Teuscher, U., Ramachandran, V. S., \& Coulson, S. (2010). Temporal sequences, synesthetic mappings, and cultural biases: The geography of time. Consciousness and Cognition, 19(1), 311320. doi:10.1016/j.concog.2010.01.003

Eagleman, D. M. (2009). The objectification of overlearned sequences: A new view of spatial sequence synesthesia. Cortex, 45(10), 1266-1277. doi:10.1016/j.cortex.2009.06.012

Mealor, A. D., Simner, J., Rothen, N., Carmichael, D. A., \& Ward, J. (2016). Different Dimensions of Cognitive Style in Typical and Atypical Cognition: New Evidence and a New Measurement Tool. PLoS One, 11(5), e0155483.

Root, N., Asano, M., Melero, H., Kim, C.-Y., Sidoroff-Dorso, A. V., Vatakis, A., . . Rouw, R. (2021). Do the colors of your letters depend on your language? Language-dependent and universal influences on grapheme-color synesthesia in seven languages. Consciousness and Cognition, 95, 103192. doi:https://doi.org/10.1016/i.concog.2021.103192

Rothen, N., Jünemann, K., Mealor, A. D., Burckhardt, V., \& Ward, J. (2016). The sensitivity and specificity of a diagnostic test of sequence-space synaesthesia. Behavior Research Methods, 48(4), 1476-1481.

Sagiv, N., Simner, J., Collins, J., Butterworth, B., \& Ward, J. (2006). What is the relationship between synaesthesia and visuo-spatial number forms? Cognition, 101, 114-128.

Seron, X., Pesenti, M., Noel, M.-P., Deloche, G., \& Cornet, J. A. (1992). Images of numbers, or "when 98 is upper left and 6 sky blue". Cognition, 44, 159-196.

Smilek, D., Callejas, A., Merikle, P., \& Dixon, M. (2006). Ovals of time: Space-time synesthesia. Consciousness and Cognition.

Ward, J., Ipser, A., Phanvanova, E., Brown, P., Bunte, I., \& Simner, J. (2018). The prevalence and cognitive profile of sequence-space synaesthesia. Consciousness and Cognition, 61, 79-93. doi:10.1016/j.concog.2018.03.012

Ward, J., Rothen, N., Chang, A., \& Kanai, R. (2017). The structure of inter-individual differences in visual ability: Evidence from the general population and synaesthesia. Vision Research, 141, 293-302. 